\title{
Recombinant FVIIIFc Versus BAY 94-9027 for Treatment of Patients with Haemophilia A: Comparative Efficacy Using a Matching Adjusted Indirect Comparison
}

\author{
Zalmai Hakimi · Elena Santagostino · Maarten J. Postma • \\ Jameel Nazir
}

Received: October 9, 2020 / Accepted: December 5, 2020 / Published online: December 30, 2020

(C) The Author(s) 2020

\section{ABSTRACT}

Introduction: Prophylaxis with recombinant factor VIII (rFVIII) is the current standard of care for haemophilia A. Several approaches have been used to extend the half-life of rFVIII to improve prophylaxis outcomes. An indirect comparison of pivotal clinical trial data was performed to evaluate the relative efficacy of two extended half-life therapies approved for the prophylactic treatment of haemophilia A: recombinant FVIII-IgG 1 Fc domain fusion protein (rFVIIIFc) and pegylated rFVIII (BAY 94-9027).

Methods: Matching-adjusted indirect comparison (MAIC) was conducted to compare the rFVIIIFC individualised prophylaxis arm of the A-LONG phase III clinical trial $(n=117)$ and the BAY 94-9027 approved dosing regimens of the

Z. Hakimi $(\bowtie) \cdot$ J. Nazir

Sobi, Stockholm, Sweden

e-mail: Zalmai.Hakimi@sobi.com

J. Nazir

e-mail: Jameel.Nazir@sobi.com

E. Santagostino

Sobi, Basel, Switzerland

e-mail: Elena.Santagostino@sobi.com

M. J. Postma

University of Groningen, Groningen,

The Netherlands

e-mail: m.j.postma@rug.nl
PROTECT VIII phase II/III study $(n=110)$. Following matching for baseline characteristics, mean annualised bleeding rate (ABR) and the proportion of patients with zero bleeds were compared for rFVIIIFc and BAY 94-9027. Additional supportive analyses comparing rFVIIIFc individualised prophylaxis and the individual prophylaxis regimens included in the PROTECT VIII group (twice weekly, and every 5 and 7 days [Q5D and Q7D]) were conducted.

Results: Mean ABR was lower in the rFVIIIFC individualised prophylaxis group versus the BAY 94-9027 pooled prophylaxis population (3.0 versus 4.9 ), providing a clinically relevant and statistically significant difference (mean difference $[\mathrm{MD}]-1.9 ; 95 \%$ confidence interval $[\mathrm{CI}]-3.5$ to -0.4$)$. A statistically significant difference in ABR was also observed for rFVIIIFc compared with BAY 94-9027 Q7D (3.2 versus 6.4; $\mathrm{MD}-3.3 ; 95 \% \mathrm{CI}-6.4$ to -0.2 ). The difference in the proportion of patients with zero bleeds between rFVIIIFc (46.5\%) and BAY 94-9027 pooled prophylaxis population (38.2\%) was not statistically significant (odds ratio 1.4; 95\% CI 0.8 to 2.5 ).

Conclusions: This indirect treatment comparison indicates a statistically significant and clinically relevant difference in ABR favouring individualised prophylaxis with rFVIIIFc versus BAY 94-9027 prophylaxis. The proportion of patients with zero bleeds was numerically greater with rFVIIIFc treatment but did not achieve statistical significance. 
Keywords: Annualised bleeding rate; BAY 94-9027; Damoctocog alfa pegol; Efmoroctocog alfa; Factor VIII-Fc fusion protein; Haematology; Haemophilia A; rFVIII; Treatment outcome

\section{Key Summary Points}

Why carry out this study?

Prophylaxis with recombinant factor VIII (rFVIII) is the current standard of care for haemophilia A; however, the short halflife of these therapies means that patients must receive frequent injections.

Extended half-life (EHL) rFVIII therapies offer the option to better tailor treatment to individuals and improve prophylaxis outcomes, while reducing injection frequency

This analysis used matching-adjusted indirect comparison (MAIC) to compare outcomes from pivotal phase III studies of two EHL products at their approved doses: recombinant FVIII-IgG 1 Fc domain fusion protein (rFVIIIFc) and pegylated rFVIII (BAY 94-9027)

\section{What was learned from the study?}

Mean annualised bleeding rate was statistically significantly lower in patients who received individualised prophylaxis with rFVIIIFc compared with patients receiving BAY 94-9027 prophylaxis (3.0 versus 4.9)

The proportion of patients with zero bleeds was numerically higher in the rFVIIIFc individualised prophylaxis group compared with the BAY 94-9027 pooled prophylaxis group (46.5\% versus $38.2 \%$ ), but this difference was not significant

This indirect treatment comparison indicates that individualised prophylaxis with rFVIIIFc is associated with clinically relevant improvements in outcomes versus BAY 94-9027

\section{DIGITAL FEATURES}

This article is published with digital features, including a summary slide, to facilitate understanding of the article. To view digital features for this article go to https://doi.org/10.6084/ m9.figshare.13332854.

\section{INTRODUCTION}

Haemophilia A is a congenital bleeding disorder characterised by insufficient levels of clotting factor VIII (FVIII). In cases of severe haemophilia A, FVIII levels are less than $1 \%$ of normal, with the risk of spontaneous bleeding into tissues, muscles, and joints, the latter leading to haemophilic arthropathy $[1,2]$. In countries with access to adequate healthcare resources, primary prophylaxis with recombinant FVIII (rFVIII) is recognised as the standard of care for the treatment of individuals with severe haemophilia A [3]. The use of rFVIII therapies reduces the incidence of joint damage, haemophilic arthropathy, loss of mobility, and the associated reduction in quality of life $[1,2]$. However, standard half-life (SHL) rFVIII therapies have a relatively short half-life (about $12 \mathrm{~h}$ ), necessitating frequent injections every 2-3 days or the use of higher doses [4], presenting a substantial burden for patients and caregivers.

Extended half-life (EHL) rFVIII therapies offer the prospect of fewer injections with more flexible dosing and, consequently, the potential to optimise treatment for individual patients. Several strategies have been employed to improve the half-life of SHL rFVIII. The EHL therapy rFVIIIFc (efmoroctocog alfa, Elocta ${ }^{\circledR}$, Swedish Orphan Biovitrum AB) covalently fuses a single rFVIII molecule to the Fc domain of $\mathrm{IgG}_{1}$, resulting in a 1.5 -fold increase in half-life versus SHL rFVIII, enabling injections to be performed every 3-5 days [5]. Use of rFVIIIFc has been associated with reduced infusion frequency [6], improved health-related quality of life [7], and cost savings [8,9]. In the phase III A-LONG study of rFVIIIFc in previously treated adolescent/adult patients, 30\% of patients achieved 5-day dosing intervals in the final 
3 months of the study [6]. In an analysis of health-related quality of life in patients enrolled in A-LONG, significant improvements over time were observed in patients who received rFVIIIFc prophylaxis [7]. A recent analysis of rFVIIIFc cost effectiveness in Italy suggested that, compared with SHL rFVIII therapies, rFVIIIFc treatment was associated with a predicted lifetime cost saving of $€ 1.3$ million and a 0.39 gain in quality-adjusted life-years per patient, driven by improved bleeding- and joint-related treatment outcomes, as well as reduced administration burden [8]. A budget impact analysis conducted in the Italian National Health System showed that the introduction of rFVIIIFc to treat haemophilia A would save approximately $€ 13$ million over 3 years [9], while a similar analysis conducted in the USA showed that use of rFVIIIFc had a modest $1.4 \%$ impact on budget over 2 years compared with SHL rFVIII therapy [10].

The phase III A-LONG study in adolescent/ adult patients [5] and the Kids A-LONG study in previously treated paediatric patients [11] have established the efficacy and safety of rFVIIIFc for the treatment of severe haemophilia A. Individualised prophylaxis with rFVIIIFc administered once or twice weekly in adults and adolescents produced a clinically meaningful reduction in annualised bleeding rate (ABR) compared with episodic treatment. Pharmacokinetic findings confirmed that rFVIIIFc had an increased half-life with an increased duration above a trough FVIII level of $1 \mathrm{IU} / \mathrm{dL}$ [5]. The efficacy and safety of rFVIIIFc in previously untreated patients (PUPs) aged less than 6 years has also been demonstrated in the recently completed PUPs A-LONG study [12], while the longer-term efficacy and tolerability of rFVIIIFc, with extended dosing intervals and low ABRs, has also been demonstrated for up to 5.9 years in the ASPIRE study [13]. rFVIIIFc is approved for the treatment and prophylaxis of bleeding in paediatric, adolescent and adult patients with haemophilia A [14, 15]. The recommended dose for long-term prophylaxis is $50 \mathrm{IU} / \mathrm{kg}$ every 3-5 days, which can be adjusted to $25-65 \mathrm{IU} / \mathrm{kg}$ on the basis of individual patient responses. Currently, rFVIIIFc is the only EHL rFVIII product approved to treat children aged up to 12 years in Europe [14].

BAY 94-9027 (damoctocog alfa pegol, JIVI ${ }^{\circledR}$, Bayer Pharma AG, Leverkusen, Germany) is a site-specific, PEGylated B-domain-deleted EHL rFVIII product with at least a 1.5-fold increased half-life compared with SHL rFVIII [16]. The safety and efficacy of BAY 94-9027 was investigated in the PROTECT VIII clinical trial, a phase II/III partially randomised study in individuals aged 12-65 years [16]. BAY 94-9027 is indicated for use in previously treated adults and adolescents with haemophilia A [17, 18]. For routine prophylaxis, the recommended dose is $45-60 \mathrm{IU} / \mathrm{kg}$ every 5 days (Q5D) $[17,18]$. This can be adjusted to $60 \mathrm{IU} / \mathrm{kg}$ every 7 days (Q7D) or $30-40 \mathrm{IU} / \mathrm{kg}$ twice weekly on the basis of patient clinical characteristics [17]. PEGylation may be associated with the development of anti-PEG immunity in some individuals, leading to potential hypersensitivity reactions [19], particularly in younger patients [20]. Concerns regarding the safety profile have led to BAY 94-9027 usage being restricted to adults and adolescents at least 12 years of age $[17,18]$. In the recently completed PROTECT VIII Kids study of BAY 94-9027, 11 children aged less than 6 years discontinued treatment because of perceived loss of efficacy and/or hypersensitivity reactions [20].

When assessing the relative efficacy of similar treatments, the preferred option would be direct comparison in a randomised clinical trial. However, in the absence of direct head-to-head trials, indirect comparison of data from clinical studies may help guide treatment decisions. Matching-adjusted indirect comparison (MAIC) is a widely used, validated method for comparing the outcomes of interventions in cases where comparative trials are not available or are difficult to perform [21].

We report here the results of applying MAIC to compare the efficacy outcomes of individualised rFVIIIFc prophylaxis in the A-LONG study with BAY 94-9027 prophylaxis in the PROTECT VIII study. 


\section{METHODS}

\section{Data Sources and Sample Selection}

The pivotal trials that provided the efficacy and safety data used to obtain marketing authorisation were used as the source of data for the comparisons performed in this analysis. The comparison of the two treatments centred on the approved dosing regimens for each product. For rFVIIIFc, patients in the non-randomised individualised prophylaxis treatment arm of the A-LONG study were included [5]. The BAY 94-9027 analysis group comprised patients enrolled in all prophylaxis treatment arms (the non-randomised twice-weekly arm, and randomised Q5D and Q7D arms) of the PROTECT VIII study [16]. These analysis populations comprise patients who received treatment with the approved dose regimens of each EHL rFVIII product. The design and results of both the A-LONG and PROTECT VIII clinical studies have been described in detail elsewhere $[5,16]$, and are described in brief below.

The A-LONG study was a phase III, open-label, multicentre, partially randomised study of rFVIIIFc in patients at least 12 years of age with severe haemophilia A [5]. Patients were assigned to one of three treatment arms: individualised prophylaxis (25-65 IU/kg every 3-5 days, $n=118)$; weekly prophylaxis (65 IU $/ \mathrm{kg} ; n=24)$; and, episodic treatment (10-50 IU $/ \mathrm{kg}, n=23)$. In the individualised prophylaxis treatment arm, each subject's pharmacokinetic parameters were used to guide individual adjustments to dosing interval and/or dose to target a steadystate trough FVIII level of 1-3 IU/dL or higher as needed to maintain good control of breakthrough bleeding. Patients who had received prophylaxis prior to study entry were enrolled into the individualised prophylaxis arm, while patients who had received episodic treatment previously were eligible for individualised prophylaxis or randomisation to one of the other two treatment arms (weekly prophylaxis or episodic treatment) on the basis of their history of bleeds in the previous 12 months.

PROTECT VIII was a phase II/III, open-label, multicentre, partially randomised study of BAY
94-9027 in patients aged between 12 and 65 years with severe haemophilia A [16]. Patients receiving prophylactic therapy at enrolment were only eligible for the prophylaxis arm, while those previously receiving ondemand therapy could choose to either continue on-demand BAY 94-9027 for 36 weeks or enter the prophylaxis arm of the study. Patients enrolled in the prophylaxis arm received BAY 94-9027 $25 \mathrm{IU} / \mathrm{kg}$ twice weekly for a 10 -week run-in period. Those experiencing more than one breakthrough bleed were assigned BAY 94-9027 30-40 IU/kg twice weekly for the duration of the study; those experiencing at most one bleed during the run-in period were randomised in a $1: 1$ ratio to receive $\mathrm{BAY}$ 94-9027 either Q5D (starting at $45 \mathrm{IU} / \mathrm{kg}$, $n=43$ ) or Q7D (fixed dose of $60 \mathrm{IU} / \mathrm{kg}, n=43$ ) for 26 weeks. In cases of inadequate bleeding control, patients in the Q5D group could increase their dose to $60 \mathrm{IU} / \mathrm{kg}$. Patients in the Q7D group were not able to increase their dose; however, they could be switched to more frequent dosing (Q5D or twice weekly).

Both the A-LONG and PROTECT VIII studies were conducted in accordance with the Declaration of Helsinki and local regulations. The protocols were approved by the authorities and the ethics committees of the respective institutions, and signed informed consent was obtained from all patients. Informed consent for this analysis was not required given the deidentified nature of the A-LONG individualised patient-level data, and the use of anonymised, previously published data from PROTECT VIII.

\section{Outcomes}

The efficacy outcomes compared were mean $\mathrm{ABR}$ and the proportion of patients with zero bleeds. These measures are both clinically relevant and widely used in haemophilia A treatment trials. The assessment period for patients in PROTECT VIII was from randomisation at week 10 of the study through to week 36 . For A-LONG, the duration of assessment corresponded to the exposure to prophylaxis. 


\section{Statistical Analysis}

MAIC was used to compare rFVIIIFc against BAY 94-9027 for the prophylactic treatment of haemophilia A according to National Institute for Health and Clinical Excellence (NICE) Decision Support Unit methodology [21]. Individual patient data from the A-LONG study were weighted to match the mean baseline characteristics of patients enrolled in the PROTECT VIII study. All data were anonymised and no information allowing individual patients to be identified was included. The following baseline variables were included for adjustment: age (mean, standard deviation [SD]); mean (SD) body mass index (BMI); mean (SD) number of bleeding events 12 months prior to randomisation; mean (SD) number of target joints per patient; percentage of patients with at least one target joint; and ethnicity (percentage of white/ Asian patients). These variables represent those known to influence ABR on the basis of the data from the A-LONG and PROTECT VIII studies $[5,16]$. The patients remaining in the A-LONG individualised prophylaxis group following matching were used in all treatment comparisons, the number of remaining patients being the effective sample size (ESS). Outcome measures were recalculated using assigned weights: mean ABR was estimated using a weighted negative binomial regression model; the odds of zero bleeds were calculated by dividing the reweighted number of patients with and without bleeding episodes.

Additional supportive analyses were performed, comparing the rFVIIIFc individualised prophylaxis group with each individual BAY 94-9027 prophylaxis regimen included in the pooled prophylaxis group (twice weekly, Q5D, Q7D) for both mean ABR and the proportion of patients with zero bleeds.

The recalculated ABR and the odds of patients with zero bleeds from A-LONG were compared statistically with observed values for BAY 94-9027 in the PROTECT VIII study. Relative treatment effects are presented as mean difference (MD) with 95\% confidence intervals (CI) for ABR and odds ratios (OR) with 95\% CI for the proportion of patients with zero bleeds. The MD was considered statistically significant if the associated 95\% CI did not contain zero, while OR values where the associated $95 \%$ CI did not include 1.0 were considered statistically significant. Statistical comparisons were conducted in R 3.5.5 (https://www.r-project.org/) with MASS package.

\section{RESULTS}

\section{Baseline Characteristics}

The population analysed included 117 patients who were assigned to receive rFVIIIFc individualised prophylaxis in A-LONG, and 110 patients who received prophylaxis with BAY 94-9027 in PROTECT VIII (twice weekly, $n=24$; Q5D, $n=43$, Q7D, $n=43$ ). Prior to matching, the mean age of the A-LONG and PROTECT VIII populations were similar (32.9 versus 34.6 years), and $68 \%$ and $66 \%$ of patients were white. In the A-LONG analysis population, $73 \%$ of patients had received prior prophylaxis, compared with $80 \%$ in the PROTECT VIII analysis population. The mean duration of treatment in the individualised prophylaxis arm of A-LONG was 32.6 weeks, and the mean study duration of PROTECT VIII was 36 weeks, which included a 10-week run-in period and the planned 26-week prophylaxis treatment period.

Individual patient data from patients enrolled in A-LONG who received individualised prophylaxis were extracted and compared with the pooled prophylaxis population of PROTECT VIII (Table 1). After matching for baseline characteristics of age, BMI, prior bleeds, target joints and ethnicity, ESS for A-LONG was $n=81$ (70\% of A-LONG population; Table 1). After matching with each of the individual prophylaxis dose groups included in the pooled prophylaxis population of PROTECT VIII, the ESS values for the individualised prophylaxis group of A-LONG were $n=62$ (versus twice weekly $n=24), n=79$ (versus Q5D $n=43$ ), and $n=45$ (versus Q7D $n=43$ ). 
Table 1 Balance of baseline characteristics and ESS following matching of A-LONG individualised prophylaxis and PROTECT VIII pooled prophylaxis groups

\begin{tabular}{|c|c|c|c|c|}
\hline & \multicolumn{2}{|l|}{ Prior to matching } & \multicolumn{2}{|c|}{ rFVIIIFc adjusted population } \\
\hline & BAY 94-9027 $(n=110)$ & $\begin{array}{l}\text { rFVIIIFc unadjusted } \\
\text { population }(n=117)\end{array}$ & Estimate (SD) & ESS, $n(\%)$ \\
\hline Mean (SD) age, years & $34.6(12.9)$ & $32.9(12.8)$ & $34.6(12.9)$ & $114.8(98.1)$ \\
\hline Mean (SD) BMI & $24.7(4.7)$ & $23.0(4.3)$ & $24.7(4.7)$ & $113.2(96.7)$ \\
\hline Mean (SD) prior bleeds & $13.2(18.0)$ & $18.3(22.3)$ & $13.2(18.0)$ & $108.0(93.1)$ \\
\hline \multicolumn{5}{|l|}{ Target joints } \\
\hline Mean (SD) target joints & $1.5(1.5)$ & $1.7(2.1)$ & $1.5(1.5)$ & $102.6(87.7)$ \\
\hline$\geq 1$ target joint, $\%$ & 73 & 61 & 73 & \\
\hline \multicolumn{5}{|l|}{ Ethnicity, \% } \\
\hline White & 66 & 68 & 66 & $116.5(99.6)$ \\
\hline Asian & 25 & 22 & 25 & \\
\hline \multicolumn{5}{|l|}{ Final adjusted population } \\
\hline Mean $(\mathrm{SD})$ age, years & & & $34.6(12.9)$ & $81.1(69.9)$ \\
\hline Mean (SD) BMI & & & $24.7(4.7)$ & \\
\hline Mean $(\mathrm{SD})$ prior bleeds & & & $13.2(18.0)$ & \\
\hline \multicolumn{5}{|l|}{ Target joints } \\
\hline Mean $(\mathrm{SD})$ target joints & & & $1.5(1.5)$ & \\
\hline$\geq 1$ target joint, $\%$ & & & 73 & \\
\hline \multicolumn{5}{|l|}{ Ethnicity, \% } \\
\hline White & & & 66 & \\
\hline Asian & & & 25 & \\
\hline
\end{tabular}

$B M I$ body mass index, ESS effective sample size, $r F V I I I F c$ recombinant factor VIII-Fc fusion protein, $S D$ standard deviation

\section{Annualised Bleeding Rate}

The mean ABR for rFVIIIFc-treated patients from the individualised prophylaxis arm of the A-LONG study population before adjustment was 2.9 versus 4.9 in the PROTECT VIII pooled prophylaxis group (MD - 2.0; 95\% CI - 3.5; - 0.4). After adjustment, mean ABR was 3.0 in the individualised prophylaxis arm of A-LONG versus 4.9 in the PROTECT VIII pooled prophylaxis group. The difference between the two treatment groups was statistically significant (MD - 1.9; 95\% CI - 3.5 to - 0.4; Fig. 1 ).

Unadjusted mean ABR values for the comparison of the rFVIIIFc individualised prophylaxis group from A-LONG with each individual dose regimen included in the PROTECT VIII pooled prophylaxis group are shown in Table 2 . Following adjustment there was a statistically significant difference in mean ABR between rFVIIIFc and BAY 94-9027 Q7D (3.2 versus 6.4; MD - 3.3; 95\% CI - 6.4 to - 0.2; Fig. 1). Mean ABR was numerically lower in the rFVIIIFc 
prophylaxis group versus BAY 94-9027 twice weekly and Q5D dose groups; however, the differences were not statistically significant (Fig. 1).

\section{Proportion of Patients with Zero Bleeds}

Prior to matching, the proportion of patients with zero bleeds in the individualised rFVIIIFc prophylaxis group was $45.3 \%$ compared with $38.2 \%$ for the PROTECT VIII study analysis group (OR 1.3; 95\% CI 0.8-2.3). Following adjustment, the proportion of rFVIIIFc-treated patients with zero bleeds was $46.5 \%$ versus $38.2 \%$ for BAY 94-9027-treated patients. This difference was not statistically significant (OR 1.4; 95\% CI 0.8-2.5; Fig. 2). Unadjusted values for the proportion of patients with zero bleeds in the rFVIIIFc individualised prophylaxis group compared with each of the dose groups

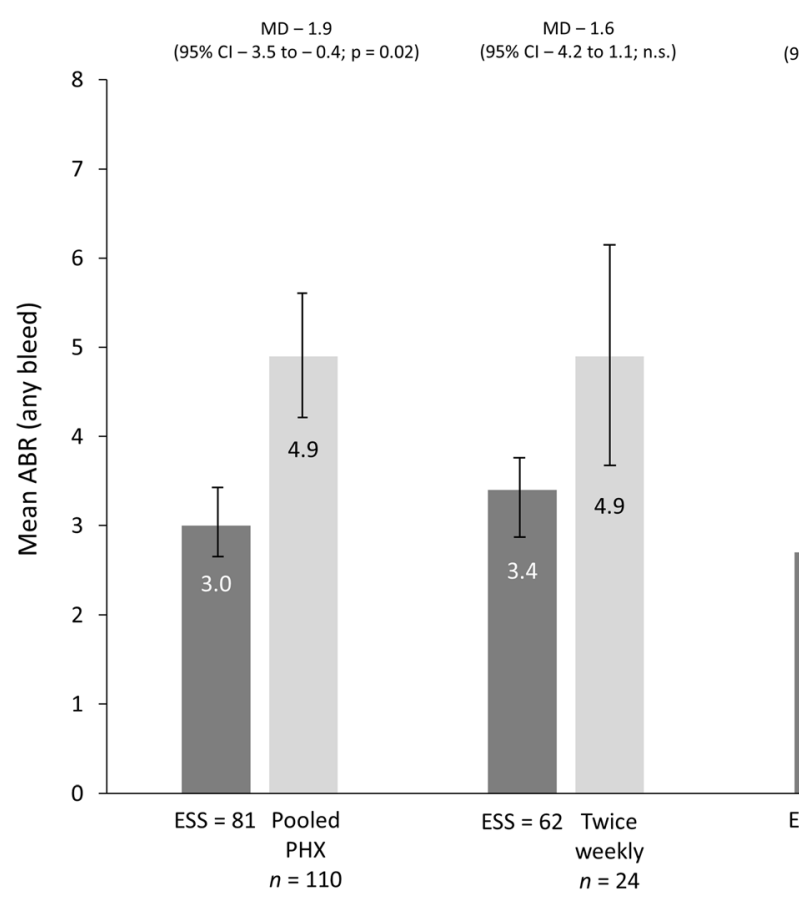

Fig. 1 ABR, for any bleeding, after matching for all baseline variables [age, BMI, percentage of patients with prior bleeds, mean number of target joints, percentage of patients with $\geq 1$ target joint, ethnicity (percentage of white and Asian patients)]. A-LONG individual prophylaxis versus PROTECT VIII pooled prophylaxis, and twice weekly, Q5D and Q7D individual dose groups. Data included in the PROTECT VIII analysis population are shown in Table 3. The proportion of rFVIIIFc-treated patients with zero bleeds was numerically greater compared with those receiving BAY 94-9027 Q5D and Q7D, and twice weekly; however, the differences were not statistically significant (Fig. 2).

\section{DISCUSSION}

This analysis, using MAIC methodology, demonstrated a lower ABR for rFVIIIFc-treated versus BAY 94-9027-treated patients and a statistically significant and clinically relevant difference. A trend towards more patients experiencing zero bleeds with rFVIIIFc treatment was also observed. The findings of the supportive analyses also indicate that prophylaxis with rFVIIIFc provides superior efficacy to BAY 94-9027 administered Q7D. The difference

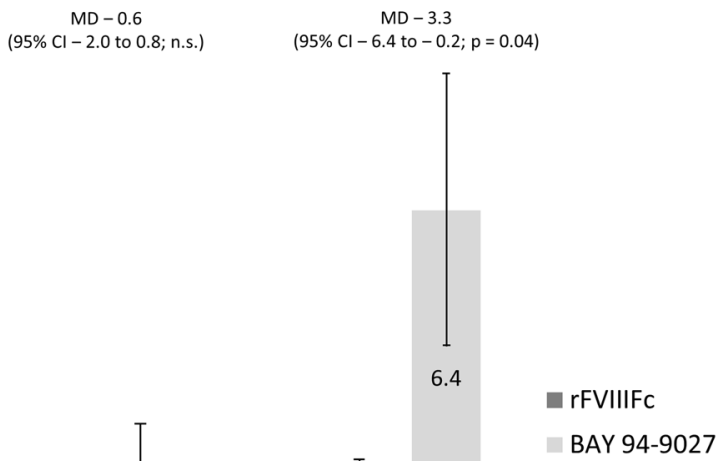

presented as mean $\pm S E$. $A B R$ annualised bleeding rate, $B M I$ body mass index, $C I$ confidence interval, $E S S$ effective sample size, $M D$ mean difference, $n$.s. not significant, $P H X$ prophylaxis, $Q 5 D$ every 5 days, $Q 7 D$ every 7 days, $r F V I I I F c$ recombinant factor VIII-Fc fusion protein, $S E$ standard error 
Table 2 Unadjusted comparison of mean ABR between the individualised rFVIIIFc prophylaxis group of A-LONG and the pooled BAY 94-9027 prophylaxis groups from PROTECT VIII

\begin{tabular}{|c|c|c|c|}
\hline \multirow[t]{2}{*}{ PROTECT VIII regimen } & \multicolumn{2}{|c|}{ Mean (SE) ABR for any bleed } & \multirow{2}{*}{$\begin{array}{l}\text { MD }(95 \% \text { CI }) \\
\text { rFVIIIFc vs BAY 94-9027 }\end{array}$} \\
\hline & BAY 94-9027 & rFVIIIFc individualised PHX & \\
\hline Q5D & $3.3(0.7)$ & & $-0.4(-1.9$ to 1.1$)$ \\
\hline Q7D & $6.4(1.5)$ & $2.9(0.4)$ & $-3.5(-6.6$ to -0.4$)$ \\
\hline Twice weekly & $4.9(1.3)$ & & $-2.0(-4.6$ to 0.6$)$ \\
\hline
\end{tabular}

$A B R$ annualised bleeding rate, $C I$ confidence interval, $M D$ mean difference, $P H X$ prophylaxis, $Q 5 D$ every 5 days, $Q 7 D$ every 7 days, $r F V I I I F c$ recombinant factor VIII-Fc fusion protein, $S E$ standard error

in efficacy between individualised prophylaxis with rFVIIIFc and BAY 94-9027 administered Q5D or twice weekly was not statistically significant, although a trend favouring rFVIIIFc was observed.

Our results differ from those of another analysis, conducted by Batt and colleagues, which used MAIC to compare the relative efficacy of BAY 94-9027 and rFVIIIFc, as well as the EHL products BAX 855 and rAHF-PFM (recombinant antihaemophilic factor plasma/albuminfree method) [22]. The results of the analysis by Batt et al. suggested no statistically significant difference in efficacy between rFVIIIFc and BAY 94-9027, assessed using both ABR and the proportion of patients with zero bleeds [22]. However, it should be noted that Batt et al. used a pooled rFVIIIFc-treated population, comprising both the individualised and weekly prophylaxis arms. Weekly prophylaxis with rFVIIIFc is not an approved therapeutic regimen [14, 15], and consequently, does not reflect use of rFVIIIFc in the clinical setting. However, Batt et al. also included a sensitivity analysis that incorporated only the rFVIIIFC individualised prophylaxis arm, the results of which showed a difference in ABR between rFVIIIFc (2.91) and BAY 94-9027 (4.25) [22]. Our findings are consistent with these results, the small differences arising from the availability of patient-level data for each analysis and differences in ESS following adjustment. Batt et al. also reported a statistically significant reduction in BAY 94-9027 utilisation versus rFVIIIFC [22]; however, the clinically relevant and statistically significant reduction in $\mathrm{ABR}$ demonstrated in our analysis suggests that any perceived benefits of reduced utilisation should be balanced against relative efficacy. We did not evaluate utilisation in our analysis, but a separate analysis assessing utilisation is planned.

In addition to the efficacy benefits observed in this analysis, rFVIIIFc offers potential safety benefits over other EHL rFVIII products. The Fc portion of rFVIIIFc is part of a naturally occurring pathway with no known inherent toxicity. In all clinical trials of rFVIIIFc, treatment was generally well tolerated, adverse events were consistent with those expected in this patient population, and no inhibitors to rFVIIIFc developed in previously treated patients $[5,11,13]$. Conversely, treatment with PEGylated BAY 94-9027 is associated with concerns around hypersensitivity reactions and lack of efficacy in paediatric patients aged up to 12 years, as a result of anti-PEG immunity $[19,20]$; BAY 94-9027 is, therefore, not approved for use in this patient population $[17,18]$. At present, rFVIIIFc is currently the only EHL product approved for use in children under 12 years of age in Europe [14].

MAIC is a validated method for the comparison of outcomes data from pivotal clinical studies where data from comparative randomised clinical trials are either not available or difficult to obtain [23], and has been accepted as a valid approach by NICE in the UK [21]. MAIC offers the opportunity to adjust for important baseline characteristics when comparing treatments, overcoming some of the methodological limitations associated with indirect treatment comparisons. Although a network meta-analysis 


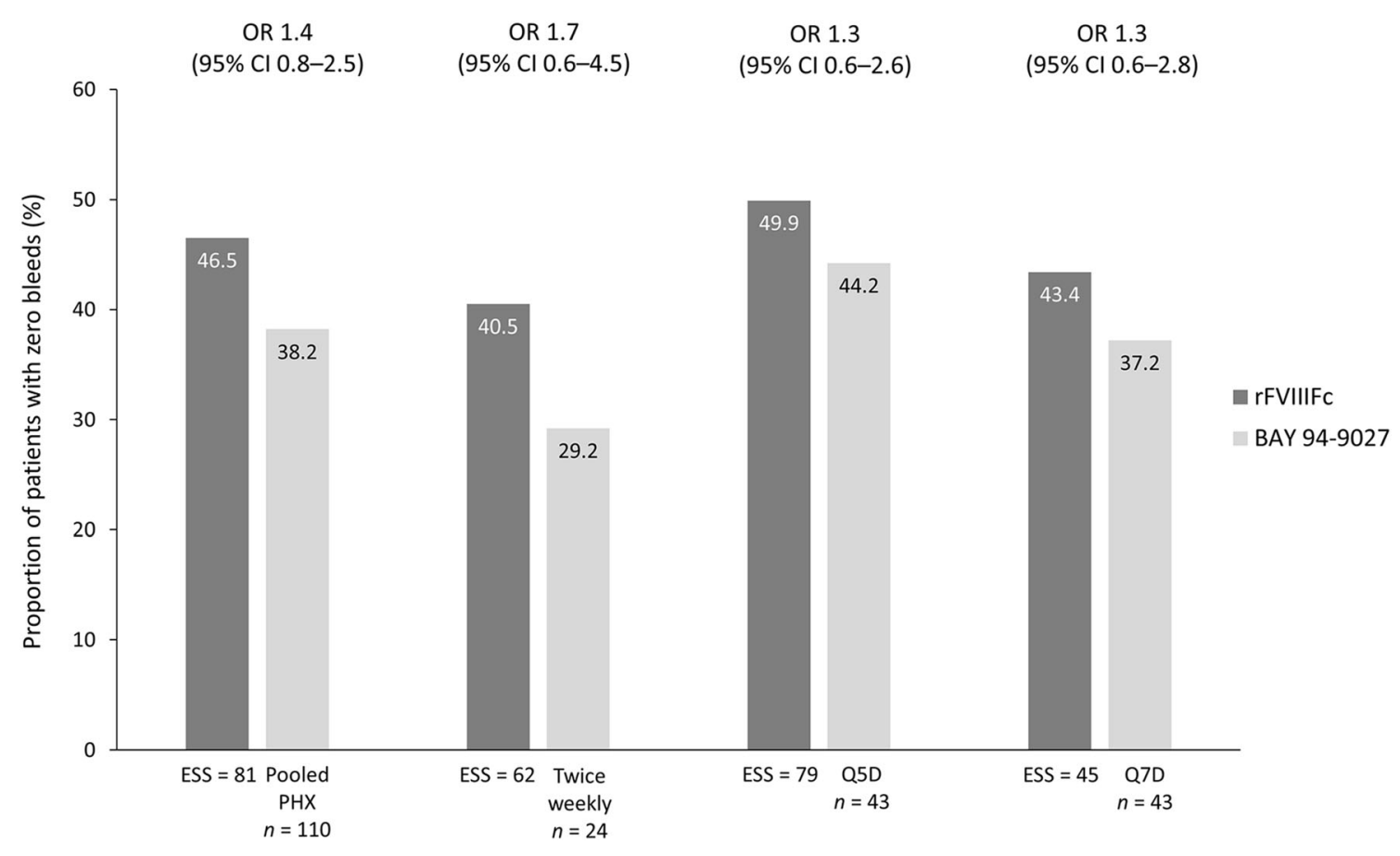

Fig. 2 Proportion of patients with zero bleeds after matching for all baseline variables [age, BMI, percentage of patients with prior bleeds, mean number of target joints, percentage of patients with $\geq 1$ target joint, ethnicity (percentage of white and Asian patients)]. A-LONG individual prophylaxis versus PROTECT VIII pooled

Table 3 Unadjusted comparison of the proportion of patients with zero bleeds between the individualised rFVIIIFc prophylaxis group of A-LONG and the pooled BAY 94-9027 prophylaxis group from PROTECT VIII

\begin{tabular}{llll}
\hline $\begin{array}{l}\text { PROTECT } \\
\text { VIII } \\
\text { regimen }\end{array}$ & $\begin{array}{l}\text { Patients with no bleeds, } \\
\%\end{array}$ & $\begin{array}{l}\text { OR }(\mathbf{9 5 \%} \\
\text { CI) }\end{array}$ \\
\cline { 2 - 3 } & $\begin{array}{l}\text { BAY } \\
\mathbf{9 4 - 9 0 2 7}\end{array}$ & $\begin{array}{l}\text { rFVIIIFc } \\
\text { individualised } \\
\text { PHX }\end{array}$ & $\begin{array}{l}\text { rFVIIIFc vs } \\
\text { BAY } \\
\mathbf{9 4 - 9 0 2 7}\end{array}$ \\
\hline Q5D & 44.2 & & $1.1(0.5-2.1)$ \\
Q7D & 37.2 & 45.3 & $1.4(0.7-2.9)$ \\
Twice weekly & 29.2 & & $2.0(0.8-5.2)$ \\
\hline
\end{tabular}

$C I$ confidence interval, $O R$ odds ratio, $P H X$ prophylaxis, Q5D every 5 days, $Q 7 D$ every 7 days, rFVIIIFC recombinant factor VIII-Fc fusion protein prophylaxis, and twice weekly, Q5D and Q7D individual dose groups. $C I$ confidence interval, $B M I$ body mass index, $E S S$ effective sample size, $O R$ odds ratio, $P H X$ prophylaxis, QSD every 5 days, Q7D every 7 days, rFVIIIFc recombinant factor VIII-Fc fusion protein

(NMA) would have enabled the comparison of more than two EHL rFVIII products, in this instance, differences in the design of the pivotal clinical studies meant that it was not possible to generate the network of direct and indirect comparisons required for an NMA [24]. Both studies had complex methodological designs, comprising both randomised and non-randomised elements. The individualised prophylaxis arm of the A-LONG study was assessed in the non-randomised element of the study, while on-demand treatment was assessed in the randomised element [5]. Conversely, the prophylaxis treatment arms used to derive the pooled prophylaxis analysis group of PROTECT VIII were assessed in the randomised element of the study, while on-demand treatment was assessed in the non-randomised element [16].

The use of data from two large phase III study populations, which are comparable in terms of documented patient baseline and 
disease characteristics, together with the use of the clinically relevant parameters, ABR and the proportion of patients with zero bleeds, contribute to the strength of this analysis. The robustness of the population analysed is further demonstrated by the relatively small observed differences between the unadjusted outcomes and the adjusted values obtained following population matching. Furthermore, while the data indicate that treatment with rFVIIIFc is associated with a numerically greater proportion of patients with zero bleeds than with BAY 94-9027, the shorter assessment period of PROTECT VIII (26 weeks) compared with that of A-LONG (32 weeks) could potentially result in an underestimate of the rFVIIIFc treatment effect. However, comparisons generated using MAIC are associated with some limitations. Although differences in published baseline data could be accounted for using MAIC, undocumented differences in baseline data could not be eliminated. Other variables, such as geographic region or FVIII genotype, were not available in PROTECT VIII and could not be used in the adjustment process. Similarly, adjustment for differences in the assessment periods of the two studies could not be included in the analysis, as these data were not available for PROTECT VIII. Furthermore, detailed clinical data for the patients in these studies are limited, and information on, for example, spontaneous bleeds such as the recently reported case of a spontaneous subepithelial haemorrhage (Antopol-Goldman lesion) in the renal pelvis and ureter of a patient with haemophilia $\mathrm{A}$ and inhibitors [25]. The absence of a placebo group in these clinical studies means that there can be no adjustment for residual confounding; other confounding factors not reported in the trials may also have an influence on the results reported here. The comparison between the rFVIIIFc individual prophylaxis group of A-LONG and the three individual BAY 94-9027 prophylaxis dose groups of PROTECT VIII resulted in reductions in ESS $(n=24$ for twice weekly; $n=43$ for both Q5D and Q7D), which should be considered when interpreting these findings. Indirect treatment comparisons, such as the one performed here, provide a useful measure of the relative efficacies of similar treatments; however, they are not replacements for comparative randomised clinical trials, and further trials are needed to confirm these findings.

\section{CONCLUSIONS}

The results of this indirect treatment comparison indicate that individualised prophylaxis with rFVIIIFc provides a statistically significant and clinically relevant benefit in mean ABR versus prophylaxis with BAY 94-9027. Although the differences between rFVIIIFc and BAY 94-9027 in the proportion of patients with zero bleeds were not significant, there was a trend towards an improved outcome with individualised rFVIIIFc prophylaxis.

\section{ACKNOWLEDGEMENTS}

Funding. This study was funded by Swedish Orphan Biovitrum AB (Sobi), Stockholm, Sweden. The Rapid Service and Open Access Fees were also funded by Sobi.

Medical Writing and Editorial Assistance. Medical writing and editorial support (in the form of writing assistance, collating author comments, assembling tables/figures, grammatical editing, fact checking, and referencing) was provided by Paul Littlebury, PhD, Bioscript Medical, Macclesfield, UK, and was funded by Sobi.

Authorship. All named authors meet the International Committee of Medical Journal Editors (ICMJE) criteria for authorship for this article, take responsibility for the integrity of the work as a whole, and have given their approval for this version to be published.

Prior Presentation. An abstract containing some of the data on which this manuscript is based was submitted to the International Society for Pharmacoeconomics and Outcomes Research (ISPOR) Europe 2020 Annual Congress 
and has been accepted for presentation as a poster (Nov 2020).

Disclosures. Zalmai Hakimi, Elena Santagostino, and Jameel Nazir are employees of Sobi. Maarten J. Postma received grants and honoraria from various pharmaceutical companies, all unrelated to this paper.

Compliance with Ethics Guidelines. Both the A-LONG and PROTECT VIII studies were conducted in accordance with the Declaration of Helsinki and local regulations. The protocols were approved by the authorities and the ethics committees of the respective institutions, and signed informed consent was obtained from all patients.

Informed consent for this analysis was not required given the deidentified nature of the A-LONG individualised patient-level data, and the use of anonymised, previously published data from PROTECT VIII.

Data Availability. The data set used and analysed during the current study are available from the corresponding author on reasonable request.

Open Access. This article is licensed under a Creative Commons Attribution-NonCommercial 4.0 International License, which permits any non-commercial use, sharing, adaptation, distribution and reproduction in any medium or format, as long as you give appropriate credit to the original author(s) and the source, provide a link to the Creative Commons licence, and indicate if changes were made. The images or other third party material in this article are included in the article's Creative Commons licence, unless indicated otherwise in a credit line to the material. If material is not included in the article's Creative Commons licence and your intended use is not permitted by statutory regulation or exceeds the permitted use, you will need to obtain permission directly from the copyright holder. To view a copy of this licence, visit http://creativecommons.org/licenses/by$\mathrm{nc} / 4.0 /$.

\section{REFERENCES}

1. Manco-Johnson MJ, Abshire TC, Shapiro AD, et al. Prophylaxis versus episodic treatment to prevent joint disease in boys with severe hemophilia. N Engl J Med. 2007;357(6):535-44.

2. Srivastava A, Santagostino E, Dougall A, et al. WFH guidelines for the management of hemophilia, 3rd edition. Haemophilia. 2020;26(Suppl 6):1-158.

3. Samuelson Bannow B, Recht M, Negrier C, et al. Factor VIII: long-established role in haemophilia A and emerging evidence beyond haemostasis. Blood Rev. 2019;35:43-50.

4. Thornburg CD, Duncan NA. Treatment adherence in hemophilia. Patient Prefer Adherence. 2017;11: 1677-86.

5. Mahlangu J, Powell JS, Ragni MV, et al. Phase 3 study of recombinant factor VIII Fc fusion protein in severe hemophilia A. Blood. 2014;123(3):317-25.

6. Shapiro AD, Ragni MV, Kulkarni R, et al. Recombinant factor VIII Fc fusion protein: extended-interval dosing maintains low bleeding rates and correlates with von Willebrand factor levels. J Thromb Haemost. 2014;12(11):1788-800.

7. Wyrwich KW, Krishnan S, Auguste P, et al. Changes in health-related quality of life with treatment of longer-acting clotting factors: results in the A-LONG and B-LONG clinical studies. Haemophilia. $2016 ; 22(6): 866-72$.

8. Bullement A, McMordie ST, Hatswell AJ, Li N, Wilson $\mathrm{K}$. Cost-effectiveness analysis of recombinant factor VIII Fc-fusion protein (rFVIIIFc) for the treatment of severe hemophilia A in Italy incorporating real-world dosing and joint health data. Pharmacoecon Open. 2020;4(1):133-42.

9. Lorenzoni V, Triulzi I, Turchetti G. Budget impact analysis of the use of extended half-life recombinant factor VIII (efmoroctocog alfa) for the treatment of congenital haemophilia A: the Italian National Health System perspective. BMC Health Serv Res. 2018;18(1):596.

10. McMullen S, Buckley B, Hall E 2nd, Kendter J, Johnston K. Budget impact analysis of prolonged half-life recombinant FVIII therapy for hemophilia in the United States. Value Health. 2017;20(1): 93-9.

11. Young G, Mahlangu J, Kulkarni R, et al. Recombinant factor VIII Fc fusion protein for the prevention and treatment of bleeding in children with severe hemophilia A. J Thromb Haemost. 2015;13(6): 967-77. 
12. Königs C OM, Dunn A, Kulkarni R, et al. Final results of PUPs A-LONG study: evaluating safety and efficacy of rFVIIIFc in previously untreated patients with haemophilia A (abstract). Res Pract Thromb Haemost. 2020;4.

13. Nolan B, Mahlangu J, Pabinger I, et al. Recombinant factor VIII Fc fusion protein for the treatment of severe haemophilia A: final results from the ASPIRE extension study. Haemophilia. 2020;26(3): 494-502.

14. ELOCTA ${ }^{\circledR}$ Summary of Product Characteristics (Internet). https://www.ema.europa.eu/en/documents/ product-information/elocta-epar-product-informa tion_en.pdf. Accessed May 2020.

15. ELOCTATE ${ }^{\circledR}$ Prescribing Information (Internet). https://www.fda.gov/media/88746/download. Accessed May 2020.

16. Reding MT, Ng HJ, Poulsen LH, et al. Safety and efficacy of BAY 94-9027, a prolonged-half-life factor VIII. J Thromb Haemost. 2017;15(3):411-9.

17. JIVI ${ }^{\mathrm{TM}}$ Summary of Product Characteristics (Internet). https://www.ema.europa.eu/en/documents/ product-information/jivi-epar-productinformation_en.pdf. Accessed May 2020.

18. JIVI ${ }^{\circledR}$ Package Insert (Internet). https://www.fda. gov/media/115934/download. Accessed May 2020.

19. Zhang P, Sun F, Liu S, Jiang S. Anti-PEG antibodies in the clinic: current issues and beyond PEGylation. J Control Release. 2016;244(Pt B):184-93.
20. Santagostino E, Kenet G, Fischer K, Biss T, Ahuja S, Steele M. PROTECT VIII Kids: BAY 94-9027 (PEGylated Recombinant Factor VIII) safety and efficacy in previously treated children with severe haemophilia A. Haemophilia. 2020;26(3):e55-65.

21. NICE DSU Technical Support Document 18: Methods for population-adjusted indirect comparisons in submission to NICE. (Internet). http://nicedsu. org.uk/wp-content/uploads/2017/05/Populationadjustment-TSD-FINAL.pdf. Accessed May 2020.

22. Batt K, Gao W, Ayyagari R, et al. Matching-adjusted indirect comparisons of annualized bleeding rate and utilization of BAY 94-9027 versus three recombinant factor VIII agents for prophylaxis in patients with severe hemophilia A. J Blood Med. 2019;10:147-59.

23. Signorovitch JE, Sikirica V, Erder $\mathrm{MH}$, et al. Matching-adjusted indirect comparisons: a new tool for timely comparative effectiveness research. Value Health. 2012;15(6):940-7.

24. Rouse B, Chaimani A, Li T. Network meta-analysis: an introduction for clinicians. Intern Emerg Med. 2017;12(1):103-11.

25. Sahin TK, Aladag E, Setterzade E, Guven GS, Haznedaroglu IC, Aksu S. Spontaneous subepithelial hemorrhage of renal pelvis and ureter (AntopolGoldman lesion) in hemophilia A patient with inhibitor: case report and review of the literature. Medicine (Baltimore). 2020;99(26):e20851. 